\title{
Performance Analysis of a Dual Cycle Engine with Considerations of Pressure Ratio and Cut-Off Ratio
}

\author{
R. EBRAHIMI* \\ Department of Agriculture Machine Mechanics, Shahrekord University, P.O. Box 115, Shahrekord, Iran
}

(Received March 14, 2010)

\begin{abstract}
The dual cycle is a better approximation to the modern high speed compression ignition engine than either the Diesel cycle or the Otto cycle. Therefore, this study is aimed at investigating the effects of pressure and cut-off ratios on the dual cycle performance with considerations of heat transfer loss, variable specific heat ratio and friction irreversible losses. By using finite-time thermodynamics theory, the relations between the power output and the compression ratio, between the thermal efficiency and the compression ratio, as well as the optimal relation between power output and the efficiency of the cycle are obtained. The results show that if compression ratio is smaller than certain value, the increase of pressure ratio and the decrease of cut-off ratio make the power output bigger. While if compression ratio exceeds certain value, the power output first increases and then starts to decline as pressure ratio and cut-off ratio are increased. With further increase in compression ratio, the power output decreases with increasing pressure ratio and decreasing cut-off ratio. The effects of pressure and cut-off ratios on the variation of the thermal efficiency with compression ratio are similar to those for the power output. The results obtained in this work can provide significant guidance for the performance evaluation and improvement of modern high speed compression ignition engines.
\end{abstract}

PACS numbers: 05.70.Ln, 82.60.Fa, 88.05.-b

\section{Introduction}

Studies on the gas cycles as the models of internal combustion engines are useful tool for illustrating some of the important parameters affecting engine performance. The Otto cycle considers one special case of an internal combustion engine, whose combustion is so rapid that the piston does not move during the combustion process and thus combustion is assumed to take place at constant volume. The Diesel cycle models another special case of an internal combustion engine whose combustion is controlled so that the beginning of the expansion stroke occurs at constant pressure. Modern compression ignition engines start to inject the fuel much earlier in the cycle, somewhere around $20^{\circ}$ before top dead center, instead of injecting the fuel late in the compression stroke near top dead center, as was done in early engines. The first fuel then ignites late in the compression stroke, and some of the combustion occurs almost at constant volume at top dead center, much like the Otto cycle [1]. The air standard cycle used to analyze this modern compression ignition engine cycle is called a dual cycle in which the heat input process of combustion can best be approxi-

\footnotetext{
* e-mail: Rahim.Ebrahimi@gmail.com,

Ebrahimi-r@agr.sku.ac.ir
}

mated by a dual process of constant volume followed by constant pressure. Therefore, the dual cycle is a better approximation to the modern high speed compression ignition engine than either the Diesel cycle or the Otto cycle $[2,3]$.

Recently, the analysis and optimization of thermodynamic cycles for different optimization objectives has made tremendous progress by using finite-time thermodynamics [4-7]. Chen [8] analyzed and optimized the power potential of a dual cycle considering the effect of combustion on power. In his work, also, he criticized the correctness of the optimum maximum temperature and the optimum cut-off ratio value. Lin et al. [9] considered the effect of heat transfer through a cylinder wall on the work output of the dual cycle. The results show that the optimum compression ratio corresponding to maximum work output decreases with the increase of the pressure ratio. Sahin et al. [10] made a comparative performance analysis of an endoreversible dual cycle under the maximum ecological function and maximum power conditions. The optimal performances and design parameters, such as compression ratio, pressure ratio and thermal conductance allocation ratio which maximize the ecological objective function are investigated. The results are compared with those of the maximum-power performance criterion. Wang et al. [11] studied the effect of pressure ratio on the efficiency versus compression ratio 
curves, power output versus compression ratio curves and power output versus efficiency curves for the dual cycle. Chen et al. [12] determined the characteristics of power and efficiency for dual cycle with heat transfers and friction losses. It is found that there are optimal values of the pressure and cut-off ratios at which the power output and efficiency attain their maxima. Hou [3] derived the performance characteristics of the dual cycle with only the heat transfer loss and studied the effects of heat transfer loss and cut-off ratio on the performance of the cycle. The effects of cut-off and pressure ratios on performance of an irreversible dual cycle were presented by Parlak et al. [13, 14]. Ust et al. [15] performed an ecological performance analysis for an irreversible dual cycle by employing the new thermo-ecological criterion as the objective function. The optimal principal engine design parameters, such as compression, pressure and cut-off ratios are presented to provide guidelines in the design procedure for dual engines. Optimum value of the cut-off ratio of the Diesel cycle is derived analytically and compared to the results of an experimental study of the low heat rejection engine by Parlak et al. [16]. Ozsoysal [17] examined the performance of an air-standard dual cycle. Thermal efficiency, irreversible work, cut-off ratio, pressure ratio and fuel conversion efficiency are obtained in terms of isentropic efficiencies, maximum and minimum temperatures, combustion efficiency and air-fuel ratio. It is also presented how the upper limit for compression ratio is evaluated by means of using some constraints for realizing the dual cycle. Valid ranges of the mentioned constants are explained.

The above studies were done without considering the variable specific heats of the working fluid. Therefore, Chen et al. [18] studied the effect of pressure ratio on dual cycle performances with considering the variable specific heat at constant pressure and constant volume of the working fluid. But, they did not study the effect of cut-off ratio on performance of dual cycle. However, it can be seen from this literature that there is a lack of information about the effects of pressure ratio on dual cycle performances.

On the basis of these research works, the aim of this paper is to model an air standard dual cycle with considerations of the variable specific heat ratio during a finite time, furthermore study the effects of pressure and cut-off ratios on the performance of an irreversible dual cycle engine.

\section{Thermodynamic analysis of a dual cycle}

The temperature-entropy $(T-S)$ diagram of an irreversible dual heat engine is shown in Fig. 1 , where $T_{1}$, $T_{2 s}, T_{2}, T_{3}, T_{4}, T_{4 s}$ and $T_{5}$ are the temperatures of the working substance in state points $1,2 s, 2,3,4,4 s$ and 5 . Process $1 \rightarrow 2 s$ is a reversible adiabatic compression, while process $1 \rightarrow 2$ is an irreversible adiabatic process that takes into account the internal irreversibility in the real compression process. The heat additions are an isochoric process $2 \rightarrow 3$ and an isobaric process $3 \rightarrow 4$. The process $4 \rightarrow 5 s$ is a reversible adiabatic expansion, while $4 \rightarrow 5$ is an irreversible adiabatic process that takes into account the internal irreversibility in the real expansion process. The heat-removing process is the reversible constant volume $5 \rightarrow 1$.
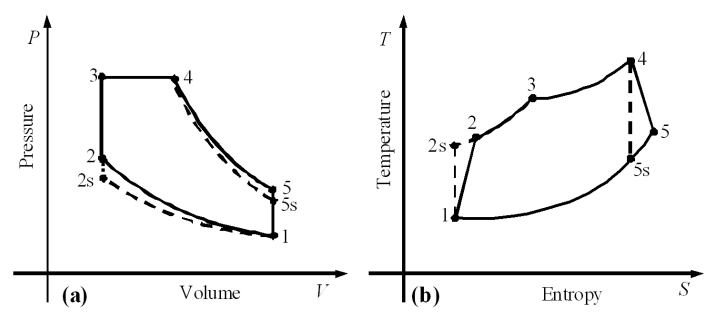

Fig. 1. (a) $P-V$ diagram, (b) $T-S$ diagram for the air standard dual cycle.

In a real cycle, the specific heat ratio is generally modeled as the first and second order equation of mean charge temperature [3, 19-21]. Thus, it can be supposed that the specific heat ratio of the working fluid is function of temperature alone and has the second order equation forms

$$
\gamma=a T^{2}+b T+c,
$$

where $\gamma$ is the specific heat ratio and $T$ is the absolute temperature. $a, b$, and $c$ are constants.

The heat added to the working fluid, during processes $(2 \rightarrow 3)$ and $(3 \rightarrow 4)$ is

$$
\begin{aligned}
\dot{Q}_{\text {in }} & =\dot{m}_{t}\left(\int_{T_{2}}^{T_{3}} c_{V} \mathrm{~d} T+\int_{T_{3}}^{T_{4}} c_{p} \mathrm{~d} T\right) \\
& =\dot{m}_{t} \int_{T_{2}}^{T_{3}}\left(\frac{R}{a T^{2}+b T+c-1}\right) \mathrm{d} T \\
& +\dot{m}_{t} \int_{T_{3}}^{T_{4}}\left(\frac{\left(a T^{2}+b T+c\right) R}{a T^{2}+b T+c-1}\right) \mathrm{d} T \\
& =\frac{2 \dot{m}_{t} R}{D}\left[\arctan \left(\frac{2 a T_{4}+b}{D}\right)-\arctan \left(\frac{2 a T_{2}+b}{D}\right)\right] \\
& +\dot{m}_{t} R\left(T_{4}-T_{3}\right),
\end{aligned}
$$

where $c_{V}$ is the constant volume specific heat for the working fluid, $c_{p}$ is the constant pressure specific heat for the working fluid, $R$ is the gas constant for the working fluid, $D$ is defined as $D=\sqrt{4 a c-4 a-b^{2}}, \dot{m}_{t}$ is the mass flow rate of the air-fuel mixture and is defined as:

$$
\dot{m}_{t}=0.5 \eta_{V} \rho_{\text {air }} V_{\mathrm{d}} N\left[1+\phi\left(m_{\mathrm{f}} / m_{\mathrm{a}}\right)_{s}\right],
$$

where $\eta_{V}$ is the volumetric efficiency, $\rho_{\text {air }}$ is the mass density of working fluid, $V_{\mathrm{d}}$ is the displaced or swept volume, $N$ is the engine speed, $\phi$ is the equivalence ratio, $m_{\mathrm{a}} / m_{\mathrm{f}}$ is the air-fuel ratio and the subscript $s$ denotes stoichiometric conditions.

The heat rejected by the working fluid during the process $(5 \rightarrow 1)$ is

$$
\dot{Q}_{\text {out }}=\dot{m}_{t} \int_{T_{1}}^{T_{5}} c_{V} \mathrm{~d} T
$$




$$
\begin{aligned}
& =\dot{m}_{t} \int_{T_{1}}^{T_{5}}\left(\frac{R}{a T^{2}+b T+c-1}\right) \mathrm{d} T=\frac{2 \dot{m}_{t} R}{D} \\
& \times\left[\arctan \left(\frac{2 a T_{5}+b}{D}\right)-\arctan \left(\frac{2 a T_{1}+b}{D}\right)\right] .
\end{aligned}
$$

According to Refs. [18, 22], the equation for a reversible adiabatic process with variable $\gamma$ can be written as follows:

$$
T V^{\gamma-1}=(T+\mathrm{d} T)(V+\mathrm{d} V)^{\gamma-1} .
$$

From Eq. (5), we get the following equation:

$$
\begin{aligned}
& \frac{1}{2(c-1)} \ln \left(\frac{a T_{j}^{2}+b T_{j}+c-1}{a T_{i}^{2}+b T_{i}+c-1}\right)+\frac{b}{(c-1) D} \\
& \times\left[\arctan \left(\frac{2 a T_{j}+b}{D}\right)-\arctan \left(\frac{2 a T_{i}+b}{D}\right)\right] \\
& -\frac{\ln \left(T_{j} / T_{i}\right)}{c-1}=\ln \left(V_{j} / V_{i}\right) .
\end{aligned}
$$

The compression, $r_{\mathrm{c}}$, pressure, $\alpha$, and $\beta$ cut-off ratios are defined as:

$$
\begin{aligned}
& r_{\mathrm{c}}=V_{1} / V_{2}, \\
& \alpha=T_{3} / T_{2},
\end{aligned}
$$

and

$$
\beta=V_{4} / V_{3}=T_{4} / T_{3} .
$$

Therefore, the equations for processes $(1 \rightarrow 2 s)$ and $(4 \rightarrow 5 s)$ are shown, respectively, by the following:

$$
\begin{aligned}
& \frac{1}{2(c-1)} \ln \left(\frac{a T_{2 S}^{2}+b T_{2 S}+c-1}{a T_{1}^{2}+b T_{1}+c-1}\right)-\frac{\ln \left(T_{2 S} / T_{1}\right)}{c-1} \\
& +\frac{b}{(c-1) D}\left[\arctan \left(\frac{2 a T_{2 S}+b}{D}\right)\right. \\
& \left.-\arctan \left(\frac{2 a T_{1}+b}{D}\right)\right]=\ln \left(1 / r_{\mathrm{c}}\right)
\end{aligned}
$$

and

$$
\begin{aligned}
& \frac{1}{2(c-1)} \ln \left(\frac{a T_{4}^{2}+b T_{4}+c-1}{a T_{5 s}^{2}+b T_{5 s}+c-1}\right)-\frac{\ln \left(T_{4} / T_{5 s}\right)}{c-1} \\
& +\frac{b}{(c-1) D}\left[\arctan \left(\frac{2 a T_{4}+b}{D}\right)\right. \\
& \left.-\arctan \left(\frac{2 a T_{5 s}+b}{D}\right)\right]=\ln \left(\frac{T_{4}}{T_{3} r_{\mathrm{c}}}\right) .
\end{aligned}
$$

The total energy of the fuel per second input into the engine can be given by $[2,23]$ :

$$
\dot{Q}_{\text {fuel }}=\eta_{\text {com }} \dot{m}_{\mathrm{f}} Q_{\mathrm{LHV}},
$$

where $\eta_{\text {com }}$ is the combustion efficiency, $Q_{\mathrm{LHV}}$ is the lower calorific value of the fuel, $\dot{m}_{\mathrm{f}}$ is the mass flow rate of the fuel and is defined as $[2,24]$ :

$$
\dot{m}_{\mathrm{f}}=0.5 \phi\left(m_{\mathrm{f}} / m_{\mathrm{a}}\right)_{s} \eta_{V} \rho_{\mathrm{air}} V_{\mathrm{d}} N \text {. }
$$

The heat loss through the cylinder wall is given in the following linear expression $[25,26]$

$$
\dot{Q}_{\mathrm{ht}}=\dot{m}_{t} B\left(T_{2}+T_{4}-2 T_{0}\right),
$$

where $B$ is constant and $T_{0}$ is the cylinder wall temper- ature.

Since the total energy of the delivered fuel $\dot{Q}_{\text {fuel }}$ is assumed to be the sum of the heat added to the working fluid $\dot{Q}_{\text {in }}$ and the heat leakage $\dot{Q}_{\mathrm{ht}}$, then

$$
\begin{gathered}
\dot{Q}_{\text {in }}=\dot{Q}_{\text {fuel }}-\dot{Q}_{\mathrm{ht}}=\eta_{\text {com }} \dot{m}_{\mathrm{f}} Q_{\mathrm{LHV}} \\
-\dot{m}_{t} B\left(T_{2}+T_{4}-2 T_{0}\right) .
\end{gathered}
$$

For the two reversible adiabatic processes $1 \rightarrow 2 s$ and $3 \rightarrow 4 s$, the compression and expansion efficiencies can be defined as [17, 24]:

$$
\eta_{\mathrm{c}}=\left(T_{2 s}-T_{1}\right) /\left(T_{2}-T_{1}\right)
$$

and

$$
\eta_{e}=\left(T_{5}-T_{4}\right) /\left(T_{5 s}-T_{4}\right) .
$$

Every time the piston moves, friction acts to retard the motion. The power lost due to friction for the Diesel modern engine is given in the following [27]:

$$
P_{\mu}=0.44\left(\frac{N}{1000}\right)^{2} \text {. }
$$

Thus, the power output of the dual cycle engine can be written as

$$
\begin{aligned}
& P_{\text {out }}=\dot{Q}_{\text {in }}-\dot{Q}_{\text {out }}-P_{\mu}=\frac{2 \dot{m}_{t} R}{D}\left[\arctan \left(\frac{2 a T_{4}+b}{D}\right)\right. \\
& +\arctan \left(\frac{2 a T_{1}+b}{D}\right)-\arctan \left(\frac{2 a T_{2}+b}{D}\right) \\
& \left.-\arctan \left(\frac{2 a T_{5}+b}{D}\right)+\frac{D}{2}\left(T_{4}-T_{3}\right)\right] \\
& -0.44\left(\frac{N}{1000}\right)^{2} .
\end{aligned}
$$

The thermal efficiency of the dual cycle engine is expressed by

$$
\begin{aligned}
\eta_{\mathrm{th}} & =\frac{P_{\mathrm{out}}}{Q_{\mathrm{in}}}=\left\{\frac { 2 \dot { m } _ { t } R } { D } \left[\arctan \left(\frac{2 a T_{4}+b}{D}\right)\right.\right. \\
+ & \arctan \left(\frac{2 a T_{1}+b}{D}\right)-\arctan \left(\frac{2 a T_{2}+b}{D}\right) \\
& \left.-\arctan \left(\frac{2 a T_{5}+b}{D}\right)+\frac{D}{2}\left(T_{4}-T_{3}\right)\right] \\
& \left.-0.44\left(\frac{N}{1000}\right)^{2}\right\} /\left\{\frac { 2 \dot { m } _ { t } R } { D } \left[\arctan \left(\frac{2 a T_{4}+b}{D}\right)\right.\right. \\
& \left.\left.-\arctan \left(\frac{2 a T_{2}+b}{D}\right)+\frac{D}{2}\left(T_{4}-T_{3}\right)\right]\right\} .
\end{aligned}
$$

When $r_{\mathrm{c}}, \eta_{\mathrm{c}}, \eta_{\mathrm{e}}$ and $T_{1}$ are given, $T_{2 s}$ can be obtained from Eq. (10), then, substituting $T_{2 s}$ into Eq. (16) yields $T_{2}$. When $\alpha$ is given, $T_{3}$ can be found from Eq. (8); then, substituting Eq. (2) into Eq. (15) yields $T_{4}$. But, when $\beta$ is given, substituting Eq. (2) into Eq. (15) yields $T_{3}$, and $T_{4}$ can be found using Eq. (9). $T_{5 s}$ can be found from Eq. (11), and $T_{5}$ can be deduced by substituting $T_{5 s}$ into Eq. (17). Substituting $T_{1}, T_{2}, T_{3}, T_{4}$ and $T_{5}$ into Eqs. (19) and (20), respectively, the power output and thermal efficiency of the dual cycle engine can be obtained. Therefore, the relations between the power output, the thermal efficiency and the compression ratio can be derived. 


\section{Results and discussion}

As it can be clearly seen from Eqs. (19) and (20), the thermal efficiency and the power output of the dual cycle are dependent on cut-off ratio and pressure ratio. The values of the constants and the parameters used in this example are summarized in Table. This study focuses on determination effects of pressure and cut-off ratio on performance of a dual cycle engine.

TABLE

Constants and parameters used in the numerical example [17-19, 28-31].

\begin{tabular}{c|c|c}
\hline \hline$a=1.6928 \times 10^{-8} \mathrm{~K}^{-1}$ & $b=-9.7617 \times 10^{-5} \mathrm{~K}^{-1}$ & $c=1.4235$ \\
$T_{0}=450 \mathrm{~K}$ & $Q_{\mathrm{LHV}}=42800 \mathrm{~kJ} \mathrm{~kg}^{-1}$ & $\phi=1$ \\
$N=3000 \mathrm{rpm}$ & $\rho_{\mathrm{air}}=1.98 \mathrm{~kg} \mathrm{~m}^{-3}$ & $\eta_{V}=0.91$ \\
$T_{1}=320 \mathrm{~K}$ & $V_{\mathrm{d}}=499.356 \mathrm{~cm}^{3}$ & $\eta_{\mathrm{com}}=0.98$ \\
$\left(m_{\mathrm{f}} / m_{\mathrm{a}}\right)_{s}=0.069$ & $\eta_{\mathrm{c}}=0.97$ & $\eta_{\mathrm{e}}=0.97$ \\
$B=0.85 \mathrm{~kJ} \mathrm{~kg}^{-1} \mathrm{~K}^{-1}$ & $\alpha=1-2$ & $\beta=1-2$
\end{tabular}

The variations in the temperatures $T_{2}, T_{3}, T_{4}$ and $T_{5}$ with the compression ratio are shown in Fig. 2 . It is found that $T_{2}, T_{3}$ and $T_{4}$ increase with increasing compression ratio, and $T_{5}$ decreases with increasing compression ratio. In Fig. 2, there are two special states: one is the state with $T_{5} \geq T_{4}$, the another is the state with $T_{3}>T_{4}$. In the two special states, the cycle cannot operate normally.

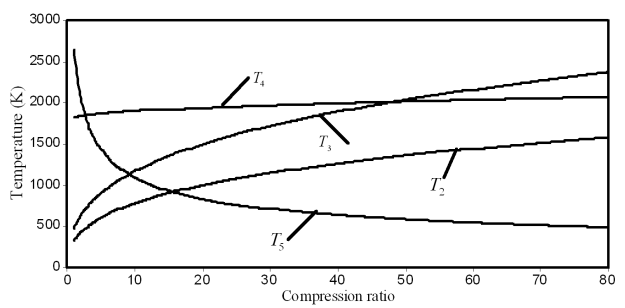

Fig. 2. The temperature versus compression ratio for $\alpha=1.5$.

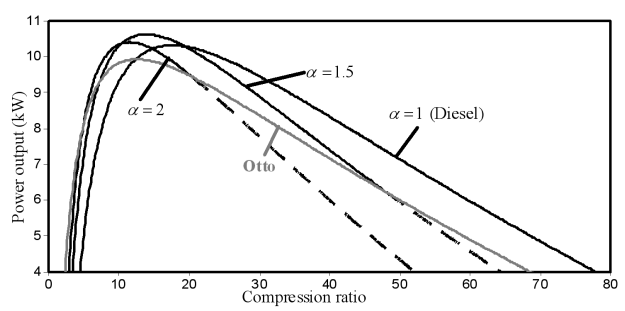

Fig. 3. Effect of pressure ratio on the variation of the power output with compression ratio.

Figures 3-8 display the influence of the parameters $\alpha$ and $\beta$ on the dual cycle performance with considerations of heat transfer and friction like term losses (the dashed lines in the figures denote where the cycle cannot work normally). The $\alpha=1$ characteristic curve corresponds to

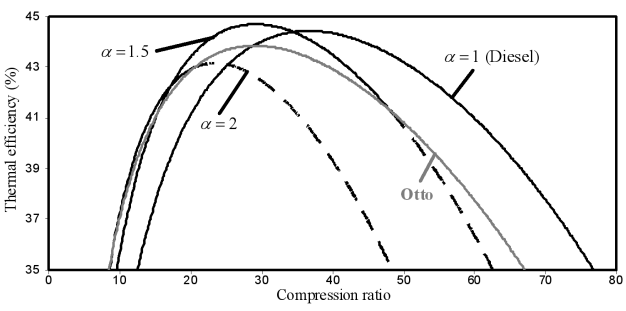

Fig. 4. Effect of pressure ratio on the variation of the thermal efficiency with compression ratio.

the Diesel cycle performance. The $\beta=1$ characteristic curve corresponds to the Otto cycle performance. From these figures, it can be found that $\alpha$ and $\beta$ play important role on the power output and the thermal efficiency. They reflect the performance characteristics of a real irreversible dual cycle engine. An increase in compression ratio first leads to an increase in power output, and after reaching a peak, the power output decreases with further increases in compression ratio. The behavior of the efficiency versus compression ratio plot is qualitatively similar to that for the power output. As can be clearly seen from these figures, the effects of $\alpha$ and $\beta$ on the power output and thermal efficiency are related to compression ratio. It can be concluded from Fig. 3 that if compression ratio is smaller than certain value, the increase of $\alpha$ makes the power output bigger. This can be attributed to the fact that the difference between heat added and heat rejected increases when $\alpha$ increases. While if compression ratio exceeds certain value, the power output first increases and then starts to decline as the $\alpha$ increases. With further increase in compression ratio, the power output decreases due to the decrease of the difference between heat added and heat rejected when $\alpha$ increases. It can be concluded from Fig. 6 that if compression ratio is less than certain value, the power output decreases with increasing $\beta$. This can be attributed to the fact that the difference between heat added and heat rejected decreases when $\beta$ increased. While if compression ratio exceeds certain value, the power output first increases and then starts to decrease as the $\beta$ increases. This, in turn, can be attributed to the fact that the difference between heat added and heat rejected first increases and then starts to decrease also with increasing $\beta$. With further increase in compression ratio, the power output increases due to the increase of the difference between heat added and heat rejected when $\beta$ increases. The behavior of the thermal efficiency with compression ratio for various pressure and cut-off ratios is similar to that for the power output. Referring to Figs. 5 and 8, it can be concluded that the maximum power output point and the maximum efficiency point are very adjacent.

Numerical calculation shows that for any same compression ratio, the smallest power output is for the Diesel cycle when $r_{\mathrm{c}} \leq 12.4$ and $r_{\mathrm{c}}>48.2$, is for the Otto cycle when $12.4<r_{\mathrm{c}} \leq 20.2$ and is for the dual cycle when $20.2<r_{\mathrm{c}} \leq 48.2$ and also the largest power output is 


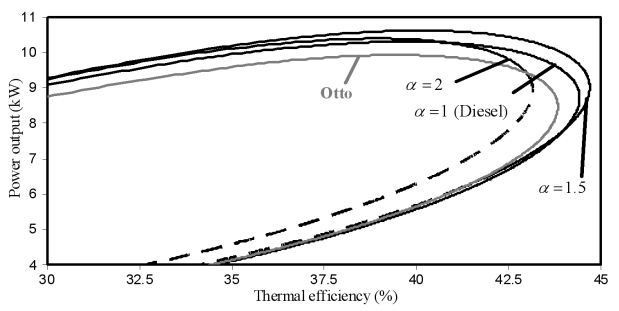

Fig. 5. Effect of pressure ratio on the variation of the power output with thermal efficiency.

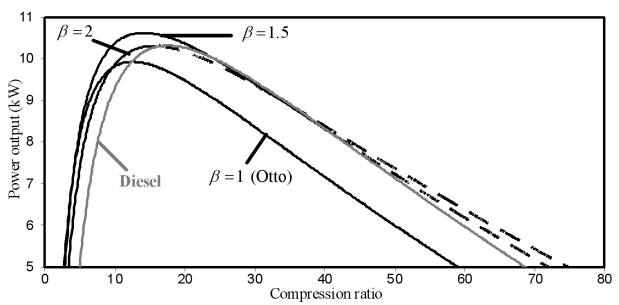

Fig. 6. Effect of cut-off ratio on the variation of the power output with compression ratio.

for the Otto cycle when $r_{\mathrm{c}} \leq 4$, is for dual cycle when $4<r_{\mathrm{c}} \leq 24$ and is for the Diesel cycle when $r_{\mathrm{c}}>24$. It can also be concluded from the numerical calculation that for any same compression ratio, the smallest thermal efficiency is for the Diesel cycle when $r_{\mathrm{c}}<28.3$ and is for the Otto cycle when $r_{\mathrm{c}} \geq 28.3$ and also the largest thermal efficiency is for the Otto cycle when $r_{\mathrm{c}} \leq 9.1$, is for dual cycle when $9.1<r_{\mathrm{c}} \leq 41$ and is for Diesel cycle when $r_{\mathrm{c}}>41$.

Referring to Figs. 3-8, it can be concluded that the maximum power output, the maximum thermal efficiency, the optimal power output corresponding to maximum thermal efficiency and optimal thermal efficiency corresponding to maximum power output increase and then start to decline with the increase of $\alpha$ and $\beta$. It can also be concluded that when $\alpha$ increases, the working range of the cycle and the optimal compression ratio corresponding to maximum thermal efficiency and the optimal compression ratio corresponding to maximum power output decrease. It can also be concluded that when $\beta$ increases, the optimal compression ratio correspond-



Fig. 7. Effect of cut-off ratio on the variation of the thermal efficiency with compression ratio.

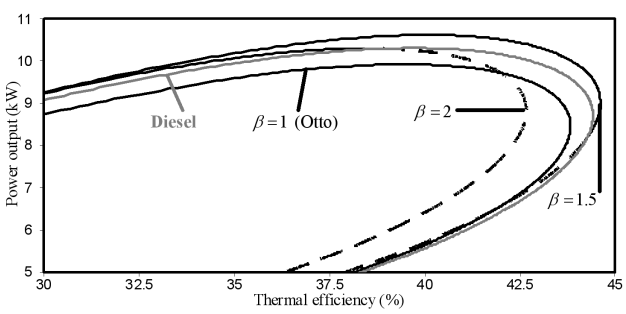

Fig. 8. Effect of cut-off ratio on the variation of the power output with thermal efficiency.

ing to maximum thermal efficiency and the optimal compression ratio corresponding to maximum power output increase, while the working range of the cycle decreases.

According to the above analysis, it can be concluded that the effects of the pressure and cut-off ratios on the cycle performance are significant. The results can provide significant guidance for the performance evaluation and improvement of real dual engines.

\section{Conclusions}

The finite-time thermodynamic model of realistic reciprocating heat-engines is a powerful tool for understanding and optimizing the performance of a reciprocating heat-engine. In this paper, an irreversible air standard dual cycle model which is more close to practice is established. In the model, the nonlinear relation between the specific heats of working fluid and temperature, the friction loss computed according to the mean velocity of the piston, the internal irreversibility described by using the compression and expansion efficiencies, and heat transfer loss are considered. The effects of pressure ratio and cut-off ratio on the power output and the thermal efficiency were analyzed by detailed numerical examples. The results show that the effects of pressure ratio and cut-off ratio on the cycle performance are obvious, and they should be considered in practical cycle analysis. The results can provide significant guidance for the performance evaluation and improvement of modern high speed compression ignition engines.

\section{Acknowledgments}

The author would like to thank the Shahrekord University for the financial support of this work.

\section{References}

[1] W.W. Pulkrabek, Engineering Fundamentals of the Internal Combustion Engine, Prentice-Hall, New Jersey 1997.

[2] J.B. Heywood, Internal Combustion Engine Fundamentals, McGraw-Hill, New York 1997.

[3] S.S. Hou, Energy Conversion Manag. 45, 3003 (2004). 
[4] G. Aragon-Gonzalez, A. Ganales-Palma, A. Leon-Galicia, J. Phys. D, Appl. Phys. 33, 1403 (2000).

[5] P.L. Curto-Risso, A. Medina, A. Calvo Hernadez, J. Appl. Phys. 105, 094904 (2009).

[6] G. Aragon-Gonzalez, A. Canales-Palma, A. Leon-Galicia, J.R. Morales-Gomez, Rev. Mex. Fis. 52, 309 (2006).

[7] L. Chen, J. Lin, C. Wu, F. Sun, Energy Conversion Manag. 39, 337 (1998a).

[8] X.Y. Chen, Energy Conversion Manag. 3, 371 (1997).

[9] J. Lin, L. Chen, C. Wu, F. Sun, Int. J. Energy Res. 23, 765 (1999).

[10] B. Sahin, U. Kesgin, A. Kodal, N. Vardar, Energy Conversion Manag. 43, 2019 (2002).

[11] W. Wang, L. Chen, F. Sun, C. Wu, Energy Int. J. 2, 340 (2002).

[12] L. Chen, F. Sun, C. Wu, Appl. Energy 79, 3 (2004).

[13] A. Parlak, Energy Conversion Manag. 46, 351 (2005).

[14] A. Parlak, B. Sahin, H. Yasar, Energy Conversion Manag. 45, 1219 (2004).

[15] Y. Ust, B. Sahin, O.S. Sogut, Appl. Energy 82, 23 (2005).

[16] A. Parlak, H. Yasar, H.S. Soyhan, C. Deniz, Energy Fuels 22, 1930 (2008).

[17] O.A. Ozsoysal, Energy Conversion Manag. 50, 2400 (2009).
[18] L. Chen, Y. Ge, F. Sun, C. Wu, Energy Conversion Manag. 47, 3224 (2006).

[19] R. Ebrahimi, J. Am. Sci. 6, 58 (2010).

[20] J.A. Gatowski, E.N. Balles, K.M. Chun, F. Nelson, J.A. Ekchian, F.B. Heywood, "A heat release analysis of engine pressure data", SAE paper 841359, 1984.

[21] M.F.J. Brunt, A.L. Emtage, "Evaluation of burn rate routines and analysis errors", SAE paper 970037, 1997.

[22] Y. Ge, L. Chen, F. Sun, C. Wu, Int. J. Ambient Energy 26, 203 (2005).

[23] R. Ebrahimi, J. Am. Sci. 6, 113 (2010).

[24] R. Stone, Introduction to Internal Combustion Engines, SAE International, 3rd ed., London 1999.

[25] Y. Ge, L. Chen, F. Sun, C. Wu, J. Energy Inst. 80 , $52(2007)$.

[26] Y. Ge, L. Chen, F. Sun, Appl. Energy 85, 618 (2008).

[27] A. Hocine, Ph.D. thesis, Université de Valenciennes et du Hainaut Cambrésis, France, 2006 (in French).

[28] R. Ebrahimi, J. Energy Inst. 83, 1 (2010).

[29] P.L. Curto-Risso, A. Medina, A. Calvo Hernadez, J. Appl. Phys. 104, 094911 (2008).

[30] Y. Ge, L. Chen, F. Sun, Math. Comput. Modelling 50, 618 (2009).

[31] R. Ebrahimi, Acta Phys. Pol. A 117, 887 (2010). 